\title{
Theoretical Research of Gas Sensing Method Based on \\ Photonic Crystal Cavity and Fiber Loop Ring-Down
}

\author{
Technique \\ Xiaolong Qian ${ }^{1}$, Yong Zhao ${ }^{1,2, *}$, Ya-nan Zhang ${ }^{1}$, Qi Wang ${ }^{1}$ \\ ${ }^{1}$ College of Information Science and Engineering, Northeastern University, Shenyang 110819, \\ China \\ ${ }^{2}$ State Key Laboratory of Synthetical Automation for Process Industries, Shenyang 110819, China \\ *Corresponding author: zhaoyong@ise.neu.edu.cn
}

\begin{abstract}
A high-sensitive gas sensing method is proposed, in which miniaturized photonic crystal (PC) cavity is utilized as the sensing element, and cryptophane E molecular is infiltrated in defected holes of PC cavity as the sensitive material to methane gas. Particularly, slow light in side-coupled PC waveguide is optimized to enhance refractive index sensitivity of the PC cavity and fiber loop ring-down technology is used to demodulate output resonant spectrum of the sensing system. Simulation results demonstrate that a refractive index sensitivity of $450 \mathrm{~nm} / \mathrm{RIU}$ and a quality factor of 1105 can be obtained in the slow light engineered PC cavity. For measurement of methane concentration, the theoretical sensitivity can be up to $4.21 \mu \mathrm{s} / \%$ and the minimum detectable concentration can be as accurate as $2.37 \mathrm{ppm}$.
\end{abstract}

Keywords: Photonic crystal cavity; slow light; fiber loop ring-down; gas concentration

$-1-$

(C) 2016. This manuscript version is made available under the Elsevier user license http://www.elsevier.com/open-access/userlicense/1.0/ 


\section{Introduction}

Gas concentration measurement has always played an important role in many industrial, biomedical and environmental applications [1]. Many methods have been proposed to measure gas concentration and among them optical gas sensor is the most popular apparatus, since they provide high sensitivity, fast response, remote monitoring, immunity to electromagnetic interference, freedom from combustion and explosion [2]. Recently, there is a growing interest in developing high-sensitive and miniaturized optical gas sensor, for implementing high-precision, low-power and portable detection of low concentration gas with fast analysis time and reduced reagent consumption [3].

\section{In recent years, photonic crystal (PC), which possesses the unique property of guiding and} manipulating light at micro-nano dimension, has become a prominent candidate for realizing miniaturized optical sensors [4]. In particular, PC cavity exhibits strong field confinement and has long photon lifetime [5]. These characteristics give rise to an optical mode with a resonant wavelength that is highly sensitive to local changes of its surrounding mediums, and make PC cavity a promising building block of high-sensitive sensors [6-11].

For gas sensor based on PC cavity, the present measurement principle is mainly based on that the resonant wavelength of PC cavity will shift with intrinsic refractive index variation of measured gas that infiltrated in the air holes of PC cavity $[10,11]$. Comparing with traditional optical gas sensor, the size of PC cavity based gas sensor can be drastically reduced. However, the corresponding refractive index variation due to concentration change is usually very low, so the designed sensor can only be used to measure the refractive index of different gases. And to enhance the measurement sensitivity, the precision fabrication of a cavity with complex and exact designed resonant structure is needed [10, 11]. Besides, as any other gases and external environment parameters can all result in comparable 
refractive index variation of air hole, they will bring many unpredictable and undesirable errors to the sensing system, and even cause the sensor unable to work. What's more, to demodulate the wavelength shift signal, an expensive optical spectrum analyzer (OSA) must be used, and the requirement of measuring condition is very strict.

Fiber loop ring-down (FLRD) technology has been widely used to demodulate the output signals of fiber Bragg grating [12, 13], long period fiber grating [14], and photonic crystal fiber interferometer [15]. In 2014, we demonstrated for the first time that the FLRD technology can also be used for high-sensitive and high-precision demodulation of the output resonant spectrum of PC cavity, which was then used for refractive index sensing [9]. Besides, we have demonstrated the feasibility of localized infiltrating different fluids into specific air holes of PC. However, to enhance the refractive index sensitivity and quality factor of PC cavity, it is needed to carefully optimize and finely tune the $\underline{\text { sizes, positions or shapes of local air holes on the order of nanometer, which will add the fabrication }}$ difficulty of the PC cavity. Besides, these optimized PC cavities based on structural adjustments are very sensitive to slight changes of structural parameters. Therefore, the nanometer-scale precision required to realize sophisticated and optimized structures eventually becomes a limiting factor in achieving desirable PC cavity [16].

Recently, it has been demonstrated that slow light in side-coupled waveguide of PC cavity can be used to enhance the refractive index sensitivity of PC cavity [17]. And the higher the group index of slow light, the higher the sensitivity of refractive index sensor is. Therefore, in this paper, to decrease the fabrication difficulty of PC cavity and its sensitivity to fabrication errors, the shapes and sizes of all air holes in PC cavity are kept the same and central positions of all air holes are periodical arranged. Then, to optimize the resonant properties of PC cavity, slow light technology is introduced in the PC 
cavity by infiltrating fluid into the first two rows of air holes adjacent to the side-coupled waveguide, without any structural variations. This post-fabrication technology can be used to modify effective refractive index of air holes after the fabrication of PC cavity, so the resonant properties can be optimized at any time, and the fabrication difficulty of PC cavity can be greatly relaxed as compared to previous optimization methods based on pure structural variations. Besides, in Ref. [9], we have demonstrated that the influences of fabrication errors on the proposed PC cavity can be calibrated.

In this paper, by using the good selectivity and high sensitivity of cryptophane E molecular to methane gas [18], a cryptophane E infiltrated PC cavity is designed to measure the concentration of methane gas. Then, to improve the system sensitivity, high group index slow light is obtained in this side-coupled PC waveguide by infiltrating fluid into the first two rows of air holes adjacent to the waveguide, without any structural variations. Finally, a fiber loop ring-down (FLRD) technology is utilized for wavelength demodulation.

\section{System Design and Sensing Principle}

Fig. 1 shows the schematic diagram of FLRD methane gas sensing system. Optical pulse was generated by modulating the output of a light source with an intensity modulator, which in turn was controlled by a signal generator. Here, the light source was a tunable laser source, with operating wavelength tuned from $1528 \mathrm{~nm}$ to $1564 \mathrm{~nm}$, and an output power of $20 \mathrm{~mW}$. Besides, an isolator was used to prevent unwanted feedback into the modulator and the light source. Then, the modulated optical pulse would be coupled into a fiber loop via a $1 \%$ arm of coupler 1 . In this fiber loop, the gas cell, as shown in the inset (a) of Fig. 1, is composed of a cryptophane E infiltrated PC cavity. The used 
1.4628 RIU. As a result, the diameter of the loop is about $50 \mathrm{~cm}$. It should be mentioned that the variation of methane gas concentration will change the refractive index of cryptophane E, and then induce transmittance variation of PC cavity at given operating wavelength. After the intensity of transmitted light changed due to different methane gas concentrations, the transmitted light would be coupled out via a $1 \%$ arm of coupler 2 , such that most of light intensity of the optical pulse would be kept inside the fiber loop and continued to travel around the fiber loop. In these continuous circulating processes, the output periodic trains of the optical pulse, which like the patterns shown in the inset (b) of Fig. 1, would be intercepted by a photodetector and converted into electrical signals. An oscilloscope was then used to view the ring-down signals. Finally, the ring-down data would be processed and displayed by a computer.

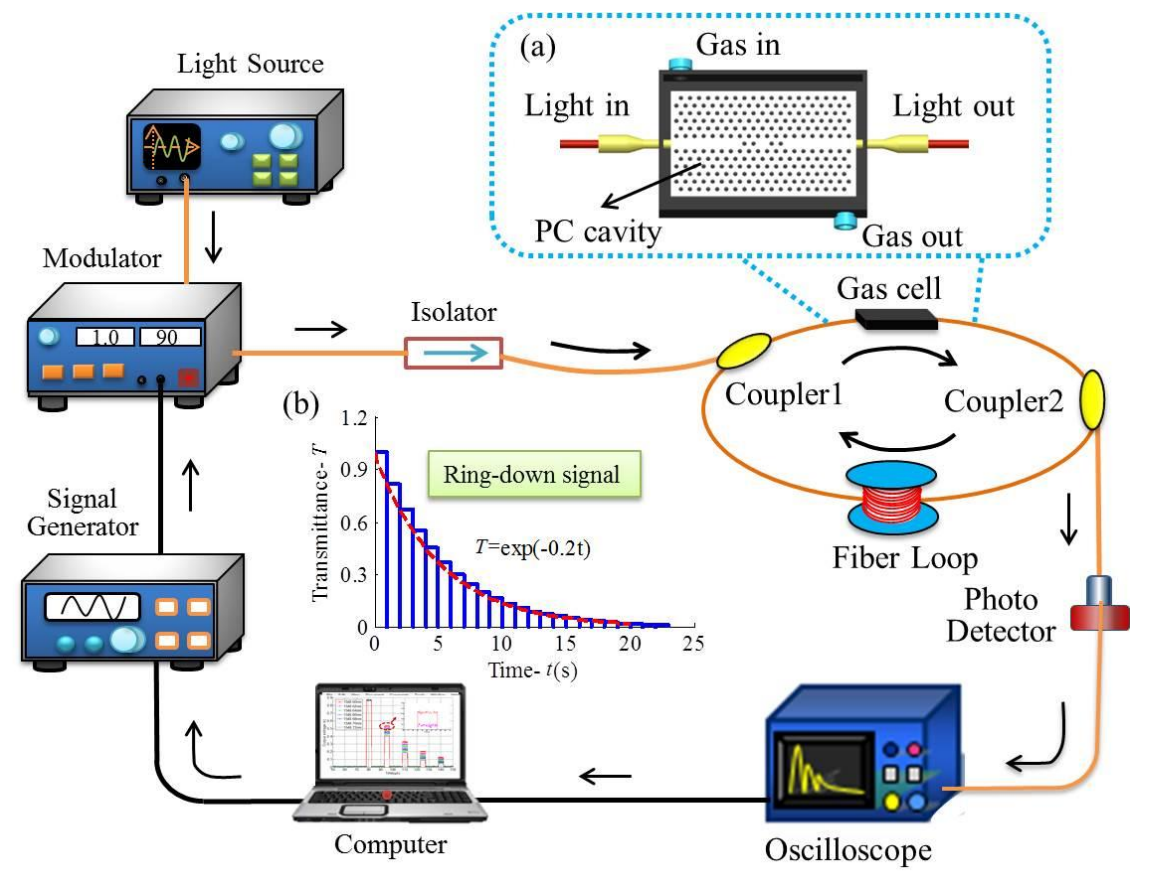

Fig. 1. Schematic diagram of FLRD methane gas sensing system, in which the inset (a) shows the specific structure of gas cell, which is composed of a cryptophane E infiltrated PC cavity, and inset (b) shows an example of ring-down signal generated by FLRD system. 
property to methane. In 2005, M. Benounis et al. demonstrated that the cryptophane E molecular possesses good adsorption and desorption properties only to alkane [18]. By using this property, they further proposed an evanescent wave methane sensor, which behaved good reversibility, repeatability and stability. However, the detection limit is still as high as $2 \% \mathrm{v} / \mathrm{v}$. Then in 2009 , S. Wu et al. fabricated a mode-filtered methane sensor, whose detection limit could be down to $0.06 \%$. However, the response time was as long as $5 \mathrm{~min}$ [19]. In this work, the cryptophane $\mathrm{E}$ molecule, which is generated by using vanillin as raw material, will be infiltrated into the defected holes of PC cavity. The stereochemical architecture and specific molecular structure of cryptophane E are shown in Fig. 2.

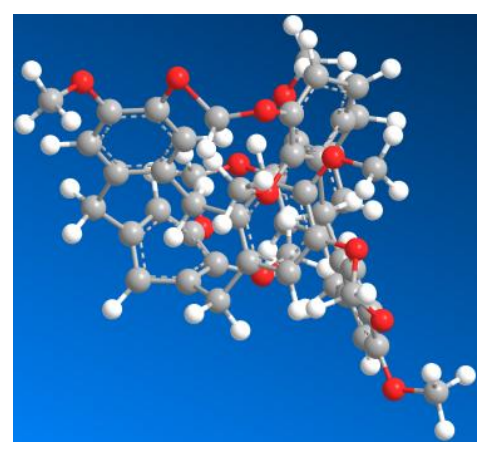

(a)

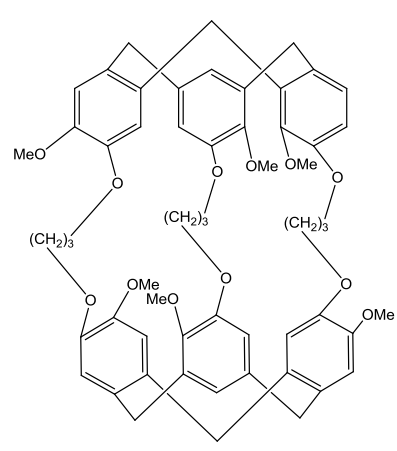

(b)

Fig. 2. Stereochemical architecture (a) and specific molecular structure (b) of cryptophane E

When methane interacts with the intra-cavity of cryptophane molecule, the corresponding dipole moment of cryptophane will be changed, along with its RI variation [18]. The relationship between refractive index $n$ of cryptophane $\mathrm{E}$ and concentration $C$ of methane gas can be given as:

$$
n=1.448-0.46 C
$$

Due to that the refractive index of cryptophane is related to the concentration $C$ of methane gas, the resonant wavelength of PC cavity will shift with the variation of $C$ (in v/v). In another point of view, the transmittance $T$ (in \%) of PC cavity at given operating wavelength is a function of $C$, which can be expressed as [9]: 


$$
\Delta T=\frac{3 \sqrt{3} Q \cdot S_{n}}{4 \lambda} \cdot \Delta n=\frac{-0.345 \sqrt{3} Q \cdot S_{n}}{\lambda} \cdot \Delta C
$$

where $\lambda$ is the resonant wavelength of PC cavity, $Q$ is the quality factor of PC cavity, $S_{n}=\Delta \lambda / \Delta n$ is the sensitivity coefficient of resonant wavelength to the refractive index $n$ of cryptophane E, and $S_{n}$ can be considered as a constant in a small range of $\Delta n$.

Since the light energy will be decreased when it transmits through the PC cavity, the transmission of optical pulse with certain wavelength will also be changed once it is sent into the fiber loop. As a result, the optical pulse will travel some round trips in the fiber loop, whose transmittance can be expressed as:

$$
I(t)=I_{0} \exp \left(-\frac{t}{\tau}\right)
$$

where $I_{0}$ and $I(t)$ denote input and output intensities of the optical pulse, respectively; $t$ is the transmission time of the optical pulse; $\tau$ represents the ring-down time, which is referred as the time taken for the light intensity decreasing to $1 / \mathrm{e}$ of the initial input light intensity and can also be expressed as [20]:

$$
\tau=-\frac{4.34 t_{r}}{\alpha}=-\frac{4.34 n_{\text {eff }} s / \mathrm{c}}{A+B}
$$

where $t_{r}=n_{e f f} s / \mathrm{c}$ is the time that the optical pulse taken to complete one ring-down trip; $s$ is the total length of fiber loop, which is set as $1 \mathrm{~km} ; \mathrm{c}=3.0 \times 10^{8} \mathrm{~m} / \mathrm{s}$ is the light velocity in vacuum; $n_{\text {eff }}$ is the effective refractive index of fiber, which equals to 1.4682 RIU; $\alpha=A+B$ is the transmission variation (in $\mathrm{dB}$ ) of total system for each round trip, where $A$ is the intrinsic loss of sensing system and $B=-10 \lg T$ is the transmission variation of PC cavity at certain working wavelength.

FLRD technology is used to measure the ring-down time $\tau$ of an optical pulse in fiber loop. So the sensitivity of the whole gas sensing system can be given as:

$$
S=\frac{\Delta \tau}{\Delta C}=\frac{\Delta \tau}{\Delta \alpha} \cdot \frac{\Delta \alpha}{\Delta T} \cdot \frac{\Delta T}{\Delta C}=\frac{-9.982 t_{r}}{\lambda T \alpha^{2}} \cdot Q S_{n}
$$


Therefore, when considering the sensing scheme of PC cavity in this design, both high $S_{n}$ and high $Q$ are desired in designing the PC cavity and PC waveguide to achieve a high sensitivity and good resolution measurement method of methane concentration. Besides, there are two advantages of this FLRD technology: one is that the output $\tau$ is independent of light intensity, resulting in a low sensitivity to loss induced by external disturbances, which can further enhance the measurement sensitivity and precision; the other one is that it needs not expensive optical spectrum analyzer and the measurement of $\tau$ for many pulses leads to a higher throughput.

\section{Modeling and Optimization}

The basic structure of our proposed PC cavity is shown in Fig. 3, in which the air holes are hole-typed and triangular arranged, with lattice constant $a$ of $320 \mathrm{~nm}$, hole radius $r$ of $99.2 \mathrm{~nm}$ and slab thickness $h$ of $220 \mathrm{~nm}$. The reasons of selecting this structure are as follows: Firstly, it is theoretically demonstrated that the width of maximum photonic band gap in a triangular lattice PC slab is larger than $\underline{\text { that of square lattice one. Also, the absolute gap of the triangular lattice PC slab is less sensitive to the }}$ variations of geometrical parameters [21]. Secondly, hole-typed PC is easy to be fabricated and its air-bridge structure is more favorable to reduce the vertical leakage of light from the substrate than pillar-typed PC in practical applications [22]. Thirdly, due to the large refractive index difference between $\mathrm{Si}$ and air, light confinement within the slab is expected to be strong. Particularly, the air-bridge silicon-on-insulator (SOI) slab composed of a Si membrane surrounded by air both above and below is one of the most popular and promising platform to implement various PC devices [6]. Considering the existing technology of SOI, the usual thickness of SOI is selected around $220 \mathrm{~nm}$, to ensure the single mode transmission of PC device. Finally, due to the scaling property of PC, the $\underline{\text { relationship between operating wavelength } \lambda \text { and lattice constant } a \text { can be expressed as: } a=U \cdot \lambda \text {, where }}$ 
$\underline{U}$ is the normalized operating frequency. Therefore, the properties of PC remain unchanged, provided

that one enlarges or reduces the structural parameters proportionally [23]. However, by considering the

tradeoff between fabrication difficult and obtained width of the photonic band gap, the radius of the

circular air hole is usually set between $0.3 a \sim 0.32 a$. Then, the corresponding normalized operating

frequency $U(=a / \lambda)$ is usually located between $0.2 \sim 0.3$. Therefore, the lattice constant $a$ is always set

around $350 \mathrm{~nm}$ to make the PC device operate around the wavelength of $1550 \mathrm{~nm}$ with low absorption

loss. Besides, the cavity length and width are selected by considering the current fabrication level. To

maintain the consistency and the repeatability of all air holes in PC, the number of air holes should be

$\underline{50 \sim 100 .}$ Therefore, the length and width of the proposed PC cavity in this paper are set as $32 \mu \mathrm{m}(100$

holes) and $16 \mu \mathrm{m}$ ( 50 holes), respectively.

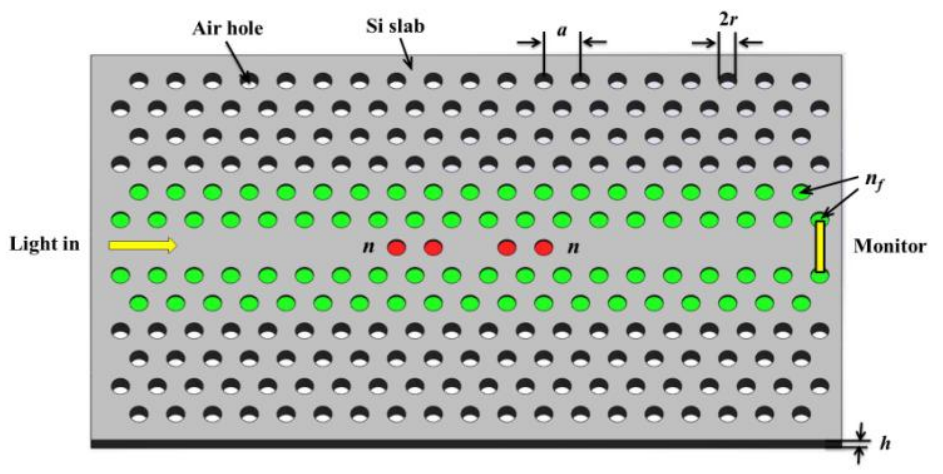

Fig. 3. Basic structure of slow light engineered PC cavity, in which the central four holes are infiltrated with cryptophane E, and the first two rows of air holes are infiltrated with suitable fluid for slow light optimization.

As for the proposed PC cavity, it is formed by the following procedures: Firstly, arranging air holes

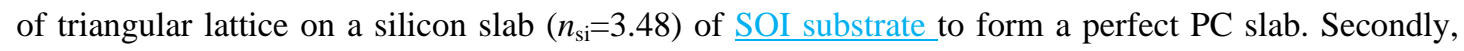
removing the central row of air holes from the perfect $\mathrm{PC}$ along the $\mathrm{x}$ direction to form a W1-typed line defected waveguide. Thirdly, inserting one pair of two air holes (defected holes) into this waveguide (considered as two reflectors), in which a point defect is introduced to form a cavity. Finally, infiltrating cryptophane $\mathrm{E}$, which is the sensitive material to methane, into the four defected holes of 
PC cavity to build the sensing region; then, infiltrating fluid with suitable refractive index into the first two rows of air holes adjacent to the waveguide to generate slow light effect. Specially, the refractive $\underline{\text { index of the first two rows of holes is defined as } n_{\mathfrak{f}} \text {, and the refractive index of the four defected holes }}$

\section{is defined as $n$.}

In the following simulations, the finite difference time domain (FDTD) method is used to analyze the field distribution and resonant properties of PC cavity, while the plane wave expansion (PWE) method is used to analyze the slow light properties of PC waveguide. Fig. 4 shows the relationship between the resonant wavelength $\lambda$ of PC cavity and the refractive index $n$ of defected holes. As shown in the inset (a) of Fig. 4, the transmission spectrum of PC cavity when $n=1.38$ behaves a narrow Lorentzian curve, whose resonant wavelength is $1451.5 \mathrm{~nm}$ and quality factor is 725 . The corresponding steady-state electric field distribution is shown in the inset (b) of Fig. 4, which demonstrates that there is a large part of light energy that confined in the point defect region. Therefore, the resonant spectrum would be sensitive to the refractive index variation of defected holes in PC cavity due to large degree of light-matter interaction inside them.

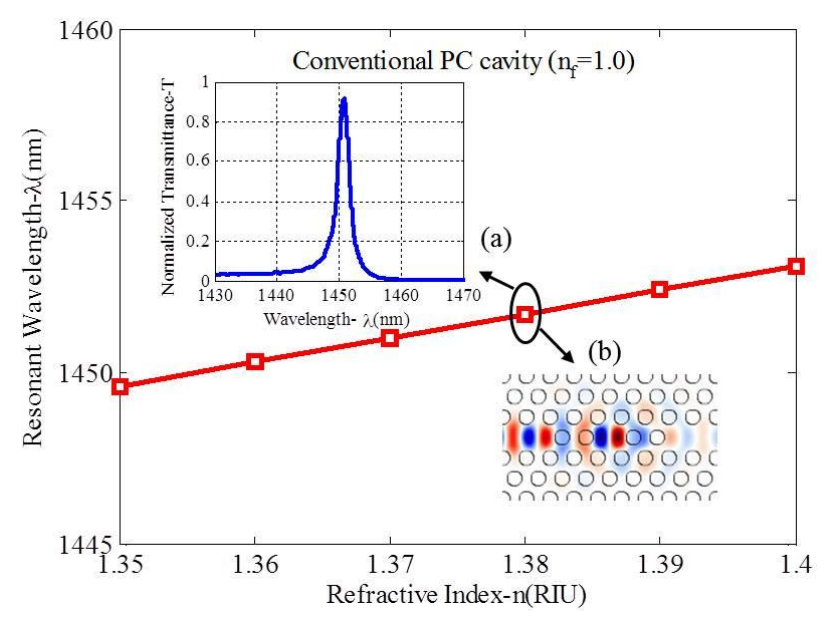

Fig. 4. Variation of resonant wavelength along with the refractive index $n$ that infiltrated in defected holes of conventional PC cavity $\left(n_{\mathrm{f}}=1.0\right)$, while the inset (a) and (b) show the transmission spectrum and corresponding steady-state electric field distribution when $n=1.38$. 
When $n$ changes from 1.33 to 1.45 with an interval of 0.02 , the resonant wavelength of PC cavity will shift to longer wavelength (red shift) with the corresponding refractive index sensitivity of $S_{n}=70$ nm/RIU. Combined with the above analyses, this property can be well used for the measurement of refractive index as well as the methane concentration. Then, to further enhance the measurement sensitivity, slow light in PC waveguide is optimized by infiltrating fluid with suitable refractive index $\underline{n_{f}}$ into the first two rows of air holes. The refractive indices of fluids that are available for infiltration span most of window, namely, from 1.33 for water based solutions to 1.5 for silica oil matching liquids and to 2.31 for Cargille liquids [24]. Considering the sensing property of PC cavity based sensor, the higher the group index of slow light, the higher the refractive index sensitivity of slow light engineered $\underline{\mathrm{PC} \text { cavity is. Besides, the wider the bandwidth of slow light, the wider the measurement range of PC }}$ cavity based sensor. Therefore, it is needed to obtain slow light with high group index and wide bandwidth. When the refractive index $n_{\mathrm{f}}$ of infiltrating fluid is changed from 1.86 to 1.90 , the corresponding slow light properties of PC waveguide are shown in Fig. 5.

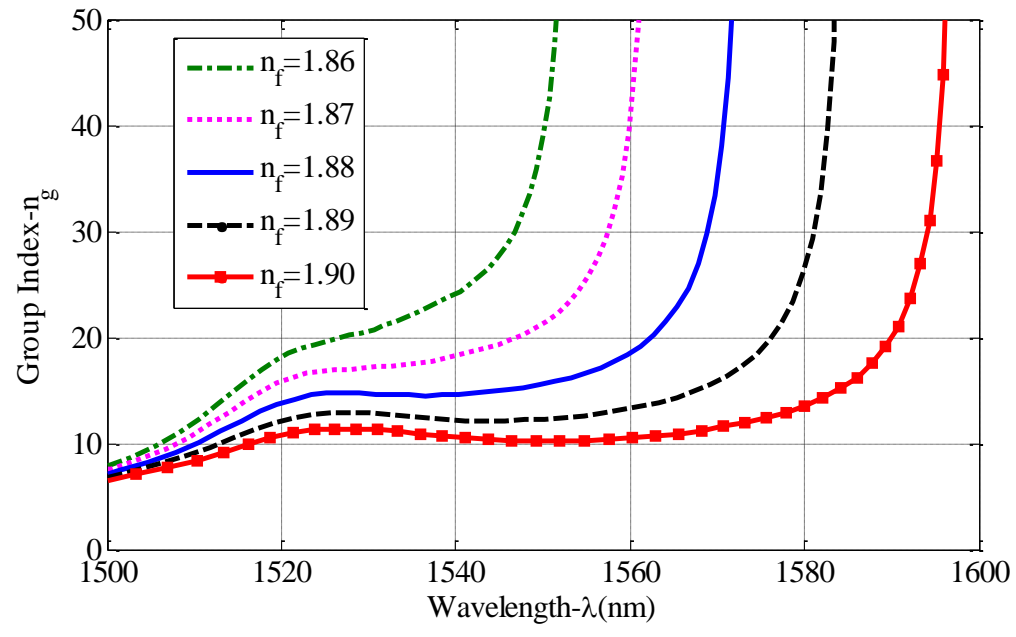

Fig. 5. Group index curves of PC waveguides with different infiltrating refractive index $n_{\underline{f}}$.

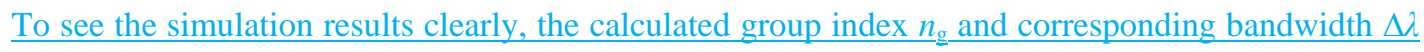
are summarized in Table I. It is shown that the group index is decreased and the corresponding 
bandwidth is increased with the increasing of infiltrating refractive index. By considering the group

index and bandwidth together, the fluid with refractive index of 1.88 is chose, which can be obtained

from the standard H series $\left(n_{\mathfrak{f}}=1.81-2.00\right)$ of Cargille Liquids. Certainly, the slow light properties of PC

waveguide can be further improved by other infiltration methods, such as infiltrating more holes and

infiltrating different fluids.

Table I Comparison of slow light properties of PC waveguide with different infiltrating refractive index

\begin{tabular}{lccccc}
\hline Refractive index $n_{\mathrm{f}}$ & 1.86 & 1.87 & 1.88 & 1.89 & 1.90 \\
\hline Group index $n_{\mathrm{g}}$ & 22 & 18 & 15.5 & 12.5 & 11 \\
Bandwidth $\Delta \lambda(\mathrm{nm})$ & 19 & 27 & 40.5 & 48 & 55 \\
\hline
\end{tabular}

Fig. 6 shows the corresponding group index and group velocity dispersion (GVD) curves of PC waveguide when fluid is infiltrated $\left(n_{\mathrm{f}}=1.88\right)$ or not $\left(n_{\mathrm{f}}=1.0\right)$. It is demonstrated that slow light with nearly constant group index of 15.5 and low GVD value on the order of $10^{6} \mathrm{ps}^{2} / \mathrm{km}$ can be realized over a bandwidth of $40.5 \mathrm{~nm}$. In this case, the variation of resonant wavelength $\lambda$ of the slow light engineered PC cavity along with the refractive index $n$ is analyzed, as shown in Fig. 7. The transmission spectrum and corresponding steady-state electric field distribution of the slow light engineered PC cavity are shown in the inset (a) and inset (b) of Fig. 7, respectively. In comparison to the case of conventional PC cavity (as shown in Fig. 4), the light energy is much strongly confined in the point defect region, which illustrates that slow light can enhance the interaction strength between light and sample that infiltrated in defected holes. In addition, the refractive index sensitivity $S_{n}$ can be increased to $450 \mathrm{~nm} / \mathrm{RIU}$, and the quality factor $Q$ is increased to 1105 . According to Eq. (5), the measurement sensitivity of PC cavity based methane sensor can be enhanced by 9.8 times after the slow light was introduced. 


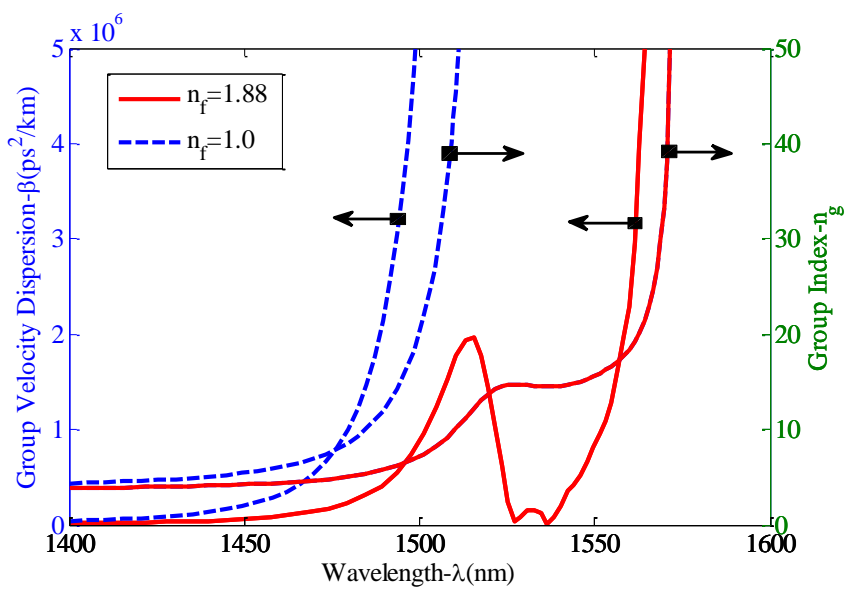

Fig. 6. Group index and GVD curves of PC waveguide when fluid is infiltrated $\left(n_{\mathrm{f}}=1.88\right)$ or not $\left(n_{\mathrm{f}}=1.0\right)$.

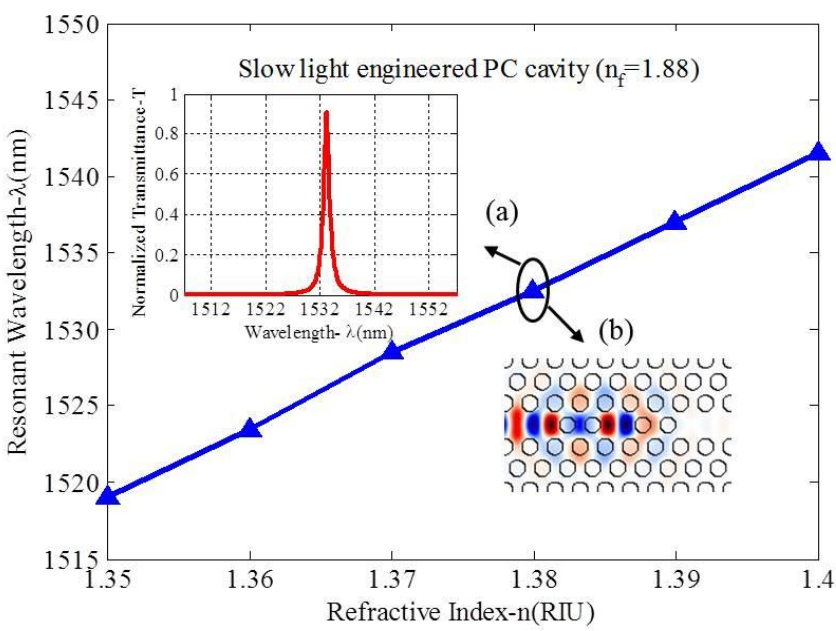

Fig. 7. Variation of resonant wavelength along with the refractive index $n$ that infiltrated in defected holes of slow light engineered PC cavity $\left(n_{\mathrm{f}}=1.88\right)$, while the inset (a) and (b) show the transmission spectrum and corresponding steady-state electric field distribution when $n=1.38$.

\section{Sensing Results and Discussion}

According to the theoretical analyses above, the normalized transmission spectra of the slow light engineered PC cavity for different methane concentrations were calculated, as shown in Fig. 8. As seen, the resonant wavelength shifts towards shorter wavelength as the methane concentration is increased from 0 to $1 \%$ with an interval of $0.2 \%$. In another point of view, if the operating wavelength of optical pulse is set to be $1563.2 \mathrm{~nm}$, where the six resonant curves are all in quasi-linear regions, the corresponding normalized intensities (marked with circle dots on the dashed line in Fig. 8) will decrease with increasing methane concentration. 


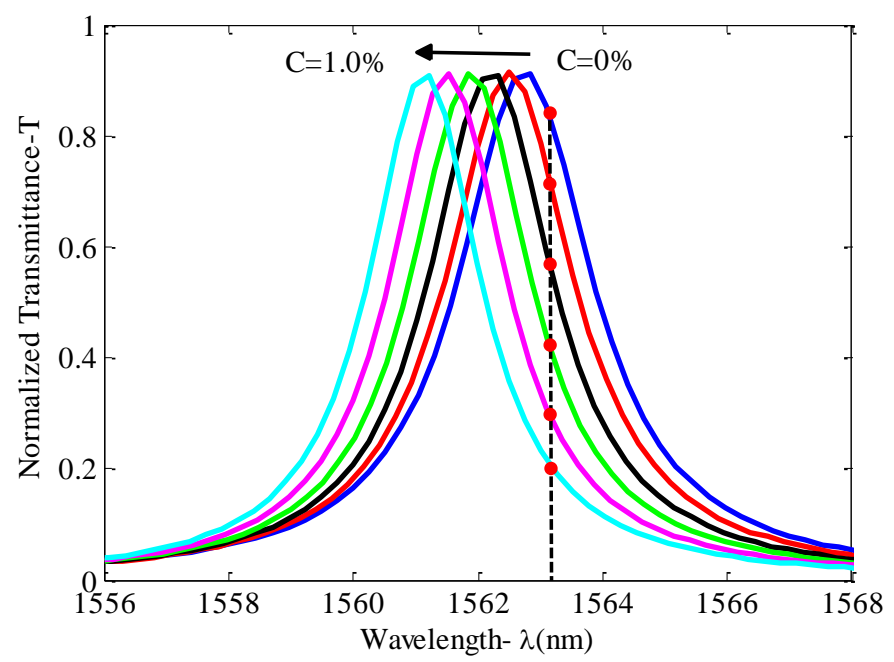

Fig. 8. Normalized transmission spectra of the slow light engineered PC cavity for different methane concentrations.

To model this relationship, the transmission loss $B$ of PC cavity at $1563.2 \mathrm{~nm}$ as a function of the methane concentration $C$ is displayed in Fig. 9. As seen, $B$ is linearly proportional to $C$ with sensitivity of $6.4 \mathrm{~dB} / \%$. According to [25] and [26], the total system loss for each round trip is about $2.51 \mathrm{~dB}$. Therefore, combined with Eq. (4), the relationship between ring-down time $\tau$ and methane concentration $C$ is also shown in Fig. 9, from which we can get that the measurement sensitivity of methane concentration can be up to $4.21 \mu \mathrm{s} / \%$.

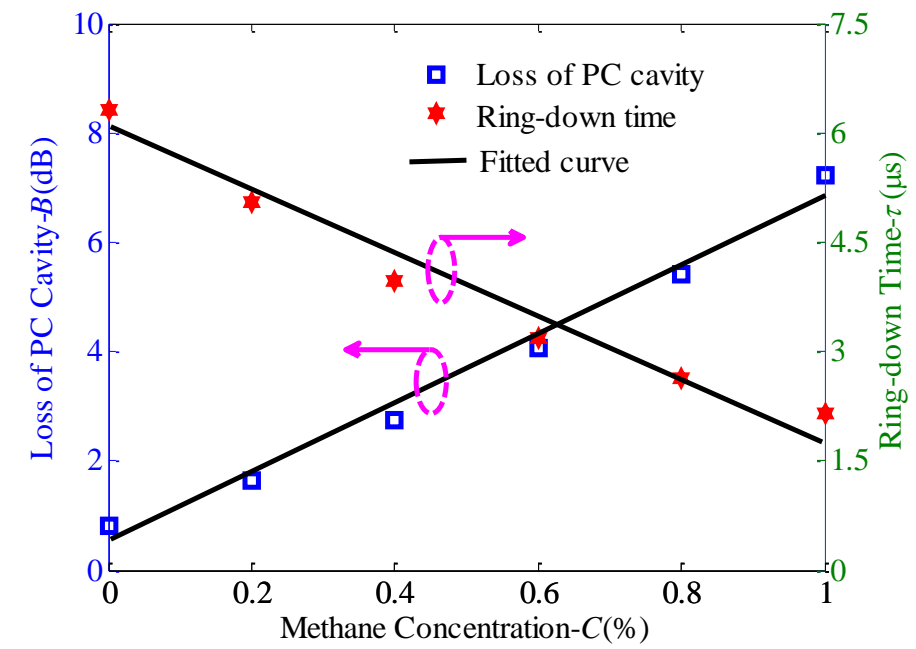

Fig. 9. Relationships of the loss of PC cavity and the corresponding ring-down time of sensing system to methane concentration. 
As the minimum detectability of a current oscilloscope can be $1 \mathrm{~ns}$, the theoretical minimum detectable methane concentration can be down to $2.37 \mathrm{ppm}$. Namely, the proposed sensing system based on PC cavity can realize the high-sensitive and high-precision measurement of gas concentration. Compared to other gas sensors based on optical technology [2], the head size of sensor (in micro scale) is greatly decreased. Besides, compared to other gas sensors based on PC cavity [10, 11], which can only identify the gas species and cannot distinguish the quantity of gas concentration, the practicability of our proposed sensor is greatly improved. Finally, compared to our published gas sensors based on slow light in PC waveguides [27, 28], whose detection limit are respectively $3.15 \mathrm{ppm}$ and $1.56 \mathrm{ppm}$, the sensing properties are equivalent. However, the used PC elements are both slotted photonic crystal waveguide, which is formed by opening a slot along the line defect of PC waveguide. However, the width and position of the introduced slot are difficult to control. Specially, in Ref. [27], the first and second rows of air holes are needed to be changed to optimize the slow light properties of PC waveguide. The above problems will all increase the fabrication difficulty of PC element. On the contrary, for the proposed PC cavity of this paper, only one kind of air hole is needed and the central positions of all air holes are periodical arranged, whose fabrication difficulty and costs can be effectively decreased.

The above analyses consider only the case that the methane concentration is changed from 0 to $1 \%$. However, the linear measurement regions and the corresponding measurement sensitivities of the sensor can be confirmed once the configuration of sensing system is fixed. Therefore, for other concentration regions, our proposed system can also work as a linear gas sensor by a premeditated calibration procedure: after the original methane concentration is calibrated, the sensor knows the original operating wavelength and sensitivity; then, the relative concentration is measured and 
accumulated according to the change of light intensity; finally, the operating wavelength and sensitivity are altered once the concentration shifts into another region.

However, when considering the practical applications of the proposed measurement method, we should consider several uncertainties: Firstly, in actual implementations, the robustness of the sensor against fabrication errors of PC is a significant factor, as the resonant property of PC cavity and the slow light property of PC waveguide are all sensitive to the structural variations of PC $[29,30]$. Fortunately, for this proposed PC cavity structure, we have demonstrated that the fabrication errors will only influence the initial resonant wavelength and will not change the refractive index sensitivity [9]. Meanwhile, by using the post-fabrication technology of fluid infiltration, the slow light property of PC waveguide allows itself to be adapted depending on actual PC structure, while the refractive index aspect offers the potential for achieving desirable slow light properties by freely choosing appropriate infiltrating fluids, which relaxes the constraint on the fabrication accuracy of PC waveguide [31]. Secondly, for the optimized structure of PC cavity with fluid infiltration, it is challenging to precisely infiltrate fluid into planned air hole whose diameter is below $200 \mathrm{~nm}$. If the refractive index of the infiltrating fluid is changed, the corresponding slow light properties of PC waveguide as well as the corresponding resonant properties of PC cavity will be changed, which will then introduce measurement error into the sensing system. However, with the development of optofluidic technology in recent years, various selective infiltration techniques for PC devices have been demonstrated by some research groups [32]-[35]. It has been demonstrated in experiment that the infiltration method with using a computer controlled micropipette actuated in three dimensions enables nearly single hole resolution [34]. Besides, in the infiltration process, it is needed to timely inspect the properties of PC 
practical situations. Therefore, the infiltration errors can be neglected for our proposed PC cavity. Thirdly, the capability to efficiently couple light from conventional fiber into the PC cavity. It remains indeed challenging because the typical waveguide cross-section ( $<1 \mu \mathrm{m}$ width and $<500 \mathrm{~nm}$ thickness) results in serious impedance mismatch phenomenon. But now many experiments have demonstrated that the coupling efficiency can be greatly improved by using suitable tapering structure [36]. Therefore, the coupling loss can now be considered as a resolved issue. Fourthly, some external parameters, such as temperature, moisture, overpressure inside the cell, will all induce the shift in the absolute resonant wavelength of PC cavity (namely, the cross sensitivity problem between these parameters), and thus influence the sensing properties of the proposed system. To avoid the temperature influence, the sample should be mounted on a temperature controlled stage inside an airtight gas cell, which allows measurements in vacuum and in different methane environments. Then, to decrease the water vapour in the gas cell, the measured gas should be dehydrated by using a freeze-dryer before entering the gas cell. Likewise, to maintain a constant gas pressure in the gas cell, the gas should be injected into the gas cell with a constant rate that just sufficient to entirely fill the gas cell, which can be determined by monitoring the shift of the resonant wavelength with increasing methane volume. When a new increase of the methane volume does not induce further shift of the resonant wavelength, the gas cell can be considered to be completely filled with the methane, whose pressure is constant. Nevertheless, the influences of these external parameters mentioned above can all be counteracted by using an identical reference system, and thus improving the measurement accuracy. However, what should be mentioned is that although many methods have demonstrated the technically feasible to resolve the above four uncertainties, they still will increase difficulties at the sensor 
fabrication. Therefore, it will take a long time to build the experimental setup and the requirement of working environment is very strict. Besides, the main work of this paper is to demonstrate the possibility of slow light in increasing the refractive index sensitivity of $\underline{\mathrm{PC} \text { cavity }}$ and fiber loop ring-down technology in enhancing the demodulation precision of resonant spectrum of $\underline{\mathrm{PC} \text { cavity. }} \underline{\text { All }}$ in all, the proposed technology opens the way for exploring miniaturized, high-sensitive and high-precision gas sensing architecture, which will offer the beneficial guidance and reference for further study. The ongoing study will focus on the experiment study of the proposed sensing method, and the experimental results will be published in the near future.

\section{Conclusion}

In summary, a gas sensing method based on photonic crystal cavity and fiber loop ring-down technique was theoretically researched. Combining the excellent resonant properties of PC cavity, the slow light enhanced light-matter interaction in PC waveguide, the good sensitivity of cryptophane E to methane gas, and the high demodulation precision and low cost of FLRD, a miniaturized, high-sensitive and high-precision gas sensing architecture was proposed, which has the potential to be used for measurements of gas concentration in some harsh environments and special conditions.

\section{Acknowledgments}

This work was supported in part by the National Science Foundation for Distinguished Young Scholars of China under Grant 61425003, the National Natural Science Foundation of China under Grant 61273059, the Fundamental Research Funds for the Central Universities under Grant N130104002, and State Key Laboratory of Synthetical Automation for Process Industries under Grant 2013ZCX09. 


\section{References:}

[1] C. Clerbaux, J. Hadji-Lazaro, S. Turquety, G. Megie, P. F. Coheur. Trace gas measurements from infrared satellite for chemistry and climate applications. Atmos. Chem. Phys., 2003, 3: 1495-1508.

[2] J. Hodgkinson, R. P. Tatam. Optical gas sensing: a review, Meas. Sci. Technol., 2013, 24(1): 012004.

[3] C. R. Englert, M. H. Stevens, C. M. Brown, J. M. Harlander, R. DeMajistre, K. D. Marr. High sensitivity trace gas sensor for planetary atmospheres: miniaturized Mars methane monitor. J. Appl. Remote Sens., 2014, 8: 083625.

[4] W. C. Lai, S. Chakravarty, Y. Zou, R. T. Chen. Silicon nano-membrane based photonic crystal microcavities for high sensitivity bio-sensing. Optics Letters, 2012, 37(7): 1208-1210.

[5] Y. Zhang, D. P. Li, C. Zeng, X. Z. Qiu, G. Gao, Y. Wang, Q. Z. Huang, J. Z. Yu, J. S. Xia. Low power and large modulation depth optical bistability in an Si photonic crystal L3 cavity. IEEE Photonics Technol. Lett., 2014, 26(23): 2399-2402.

[6] C. Caer, S. F. Serna-Otalvaro, W. W. Zhang, X. Le Roux, E. Cassan. Liquid sensor based on high-Q slot photonic crystal cavity in silicon-on-insulator configuration. Opt. Lett., 2014, 39(20): $5792-5794$.

[7] Y. Yang, H. P. Tian, D. Q. Yang, N. N. Wu, J. Zhou, Q. Liu, Y. F. Ji. Nanomechanical three dimensional force photonic crystal sensor using shoulder-coupled resonant cavity with an inserted pillar. Sens. Actuator A-Phys., 2014, 209: 33-40.

[8] Y. Zou, S. Chakravarty, D. N. Kwong, W. C. Lai, X. C. Xu, X. H. Lin, A. Hosseini, R. T. Chen. Cavity-waveguide coupling engineered high sensitivity silicon photonic crystal microcavity biosensors with high yield. IEEE J. Sel. Top. Quantum Electron., 2014, 20(4): 6900710. 
[9] Y. N. Zhang, Y. Zhao, D. Wu, Q. Wang. Fiber loop ring-down refractive index sensor based on high-Q photonic crystal cavity. IEEE Sens. J., 2014, 14(6): 1878-1885.

[10] T. Sünner, T. Stichel, S. -H. Kwon, T. W. Schlereth, S. Höfling, M. Kamp, A. Forchel. Photonic crystal cavity based gas sensor. Appl. Phys. Lett., 2008, 92(26): 261112.

[11] J. Jagerska, H. Zhang, Z. L. Diao, N. Le Thomas, R. Houdrem. Refractive index sensing with an air-slot photonic crystal nanocavity. Opt. Lett., 2010, 35(15): 2523-2525.

[12] S. Silva, D. J. Passos, M. B. Marques, O. Frazao. Chirped fiber Bragg grating cavity ring-down for strain sensing using an OTDR. Microw. Opt. Technol. Lett., 2015, 57(6): 1442-1444.

[13] H. Waechter, J. Litman, A. H. Cheung, J. A. Barnes, H. P. Loock. Chemical sensing using fiber cavity ring-down spectroscopy. Sensors, 2010, 10(3): 1716-1742.

[14] J. A. Bames, R. S. Brown, A. H. Cheung, M. A. Dreher, G. Mackey, H. P. Loock. Chemical sensing using a polymer coated long-period fiber grating interrogated by ring-down spectroscopy. Sens. Actuator B-Chem., 2010, 148(1): 221-226.

[15] W. C. Wong, W. J. Zhou, C. C. Chan, X. Y. Dong, K. C. Leong. Cavity ringdown refractive index sensor using photonic crystal fiber interferometer. Sens. Actuator B-Chem., 2012, 161(1): $108-113$

[16] T. Asano, B. S. Song, S. Noda. Analysis of the experimental Q factors ( 1 million) of photonic crystal nanocavities. Opt. Express, 2006, 14(5): 1996-2002.

[17] W. C. Lai, S. Chakravarty, Y. Zou, Y. Guo, R. T. Chen. Slow light enhanced sensitivity of resonance modes in photonic crystal biosensors, Appl. Phys. Lett., 2013, 102(4): 041111.

[18] M. Benounis, N. Jaffrezic-Renault, J. Dutasta, K. Cherif, A. Abdelghani. Study of a new evanescent wave optical fibre sensor for methane detection based on cryptophane molecules. Sens. 
Actuator B-Chem., 2005, 107(1): 32-39.

[19] S. Wu, Y. Zhang, Z. Li, S. Shuang, C Dong, M Choi. Mode-filtered light methane gas sensor based on cryptophane A. Anal. Chim. Acta, 2009, 633(2): 238-243.

[20] N. Ni, C. C. Chan, L. Xia, P. Shum. Fiber cavity ring-down refractive index sensor. IEEE Photonics Technol. Lett., 2008, 20(13-16): 1351-1353.

[21] C. G. Bostan, R. M. de Ridder. Design of photonic crystal slab structures with absolute gaps in guided modes. J. Optoelectron. Adv. Mater., 2002, 4(4): 921-928.

[22] S. G. Johnson, P. R. Villeneuve, S. H. Fan, J. D. Joannopoulos. Linear waveguides in photonic-crystal slabs. Phys. Rev. B, 2000, 62(12): 8212-8222.

[23] J. D. Joannopoulos, S. G. Johnson, J. N. Winn, R. D. Meade. Photonic crystals: molding the flow of light. 2nd ed., Princeton, 2008.

[24] http://www.cargille.com/opticalintro.shtml, "Introduction to Optical Liquids" (2011).

[25] Y. J. Jiang, J. L. Zhao, D. X. Yang, D. Q. Tang. High-sensitivity pressure sensors based on mechanically induced long-period fiber gratings and fiber loop ring-down. Opt. Commun., 2010, 283(20): 3945-3948.

[26] C. Y. Lin, X. L. Wang, S. Chakravarty, B. S. Lee, W. C. Lai, R. T. Chen. Wideband group velocity independent coupling into slow light silicon photonic crystal waveguide. Appl. Phys. Lett., 2010, 97(18): 183302.

[27] Y. N. Zhang, Y. Zhao, Q. Wang. Optimizing the slow light properties of slotted photonic crystal waveguide and its application in a high-sensitivity gas sensing system, Meas. Sci. Technol., 2013, 24(10): 105109.

[28] Y. N. Zhang, Y. Zhao, Q. Wang. Multi-component gas sensing based on slotted photonic crystal 
waveguide with liquid infiltration, Sens. Actuator B-Chem., 2013, 184: 179-188.

[29] H. Hagina, Y. Takahashi, Y. Tanaka, T. Asano, S. Noda. Effects of fluctuation in air hole radii and positions on optical characteristics in photonic crystal heterostructure nanocavities. Phys. Rev. B., 2009, 79(8): 085112.

[30] J. Liang, L. Ren, M. Yun, X. Wang. Wideband slow light with ultralow dispersion in a W1 photonic crystal waveguide. Appl. Optics, 2011, 50(31): G98-G103.

[31] Y. Zhao, Y. N. Zhang, Q. Wang, Optimization of slow light in slotted photonic crystal waveguide with liquid infiltration, J. Lightwave Technol., 2013, 31(14): 2448-2454.

[32] D. Psaltis, S. R. Quake, C. Yang. Developing optofluidic technology through the fusion of microfluidics and optics. Nature, 2006, 442(7101): 381-386.

[33] D. Erickson, T. Rockwood, T. Emery, A. Scherer, D. Psaltis. Nanofluidic tuning of photonic crystal circuits. Opt. Lett., 2006, 31(1): 59-61.

[34] F. Intonti, S. Vignolini, V. Turck, M. Colocci, P. Bettotti, L. Pavesi, S. L. Schweizer, R. Wehrspohn, D. Wiersma. Rewritable photonic circuits. Appl. Phys. Lett., 2006, 89(21): 211117.

[35] A. Casas-Bedoya, P. Domachuk, C. Grillet, C. Monat, E. C. Mägi, E. Li, B. J. Eggleton. Reconfigurable photonic crystal waveguides created by selective liquid infiltration. Opt. Express, 2012, 20(10): 11046-11056.

[36] Y. Zhao, Y. N. Zhang, Q. Wang, H. F. Hu. Review on the optimization methods of slow light in photonic crystal waveguide. IEEE Trans. Nanotechnol., 2015, 14(3): 407-426. 


\section{Biography:}

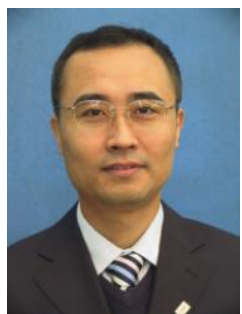

Yong Zhao received his M.A. and Ph.D. degrees, respectively, in precision instrument \& automatic measurement with laser and fiber-optic techniques from the Harbin Institute of Technology, China, in 1998 and 2001. He was awarded a first prize scholarship in 2000 by the China Instrument and Control Society and the Sintered Metal Corporation (SMC) scholarship in Japan. He was a scholarship in Japan. He was a postdoctor in the Department of Electronic Engineering of Tsinghua University from 2001 to 2003, and then worked as an associate professor in the Department of Automation, Tsinghua University of China. In 2006, he was a visiting scholar of University of Illinois in Urbana and Champagne, USA. In 2008, he was awarded as the "New Century Excellent Talents in University" by the Ministry of Education of China. In 2009, he was awarded as the "Liaoning Bai-Qian-Wan Talents" by Liaoning Province. In 2011, he was awarded by the Royal Academy of Engineering as an academic researcher of City University London. He was awarded by the National Science Foundation for Distinguished Young Scholars of China, in 2014. Now he is working in Northeastern University as a full professor. As a leader of his research group, his current research interests are the development of fiber-optic sensors and device, fiber Bragg grating sensors, novel sensor materials and principles, slow light and sensor technology, optical measurement technologies. He has authored and co-authored more than 180 scientific papers and conference presentations, 7 patents, and 4 books. He is a member in the Editorial Boards of the international journals of Sensor Letters, Instrumentation Science \& Technology, Journal of Sensor Technology, and Advances in Optical Technologies.

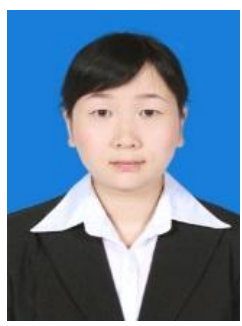

Ya-nan Zhang was born in Anhui, China, in June 1989. She received her B.A. and M.A. degrees, respectively, in 2010 and 2012 from the College of Information Science and Engineering, Northeastern University, Shenyang, China, where she is currently working toward the Ph.D. degree. Her research interests include fiber optical sensors, photonic crystal waveguide sensors, and slow light technology and its sensing applications. She has authored and co-authored more than 30 scientific papers and conference presentations.

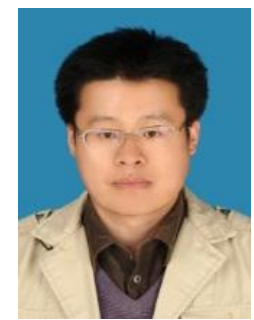

Qi Wang was born in Liaoning, China, in 1982. He received his Ph.D. degree in 2009 from the School of Physics and Optoelectronic Technology, Dalian University of Technology (DUT), Dalian, China. He is currently working in the College of Information Science and Engineering at Northeast University, China. His research interests are new photonic devices, fiber-optic sensors, optoelectronic measurement technology and system, and their industrial applications. He has authored and co-authored more than 30 scientific papers, patents and conference presentations. 